\title{
COVID-19 e repercussões na segurança alimentar e nutricional no Brasil
}

\author{
Rita de Cássia Ribeiro Silva, ${ }^{1}$ Marcos Pereira, ${ }^{2}$ Erika dos Santos Aragão, ${ }^{3}$ Jane \\ Mary de Medeiros Guimarães, ${ }^{4}$ Andrêa Jacqueline Fortes Ferreira, ${ }^{5}$ Aline dos \\ Santos Rocha, ${ }^{6}$ Natanael Jesus Silva, ${ }^{7}$ Camila Silveira Silva Teixeira, ${ }^{8}$ Ila Falcão, ${ }^{9}$ \\ Enny Santos da Paixão, ${ }^{10}$ Sandra Maria Chaves Santos, ${ }^{11}$ Tereza Campello, ${ }^{12}$ \\ Maurício Lima Barreto ${ }^{13}$
}

1 Nutricionista, mestra em Nutrição Humana Aplicada pela Universidade de São Paulo (USP) e doutora em Saúde Coletiva pelo Instituto de Saúde Coletiva (ISC) da Universidade Federal da Bahia (UFBA). Professora titular da UFBA e pesquisadora do Centro de Integração de Dados e Conhecimentos para Saúde (Cidacs) da Fundação Oswaldo Cruz (Fiocruz) da Bahia.

2 Nutricionista, mestre em Alimentos, Nutrição e Saúde pela Escola de Nutrição da Universidade Federal da Bahia (UFBA) e doutor em Saúde Pública pelo Instituto de Saúde Coletiva (ISC) da UFBA. Professor adjunto do ISC da UFBA.

3 Economista, mestra em Economia pela UFBA e doutora em Saúde Coletiva pelo Instituto de Saúde Coletiva (ISC) da Universidade Federal da Bahia (UFBA). Professora adjunta do ISC da UFBA e pesquisadora colaboradora do Centro de Integração de Dados e Conhecimentos para Saúde (Cidacs) da Fundação Oswaldo Cruz (Fiocruz) da Bahia.

4 Economista, doutora em Saúde Pública pelo Instituto de Saúde Coletiva (ISC) da Universidade Federal da Bahia (UFBA). Pesquisadora visitante da London School of Hygiene and Tropical Medicine. MBA em Economia e ATS. Pesquisadora do Programa de Economia da Saúde (PECS) do ISC da UFBA. Professora da Universidade Federal do Sul da Bahia (UFSB).

5 Nutricionista e mestra em Alimentos, Nutrição e Saúde pela Escola de Nutrição da Universidade Federal da Bahia UFBA. Doutora em Saúde Pública pelo Instituto de Saúde Coletiva (ISC) da UFBA. Pesquisadora do Centro de Integração de Dados e Conhecimentos para Saúde (Cidacs) da Fundação Oswaldo Cruz (Fiocruz) da Bahia.

6 Nutricionista pela Universidade Federal do Recôncavo da Bahia (UFRB), mestra e doutoranda pelo Programa de Pós-Graduação em Alimentos Nutrição e Saúde, da Escola de Nutrição da UFBA e pesquisadora do Centro de Integração de Dados e Conhecimentos para Saúde (Cidacs) da Fundação Oswaldo Cruz (Fiocruz) da Bahia.

7 Nutricionista pela Universidade Federal de Sergipe (UFS). Mestre em Saúde Comunitária pelo Instituto de Saúde Coletiva (ISC) da Universidade Federal da Bahia (UFBA). Atualmente, pesquisador do Centro de Integração de Dados e Conhecimentos para Saúde (Cidacs) da Fundação Oswaldo Cruz (Fiocruz) da Bahia.

8 Nutricionista, mestra em Saúde Pública pela Universidade Federal do Ceará (UFCE). Doutora em Saúde Pública pelo Instituto de Saúde Coletiva (ISC) da Universidade Federal da Bahia (UFBA) e pesquisadora do Centro de Integração de Dados e Conhecimentos para Saúde (Cidacs) da Fundação Oswaldo Cruz (Fiocruz) da Bahia.

9 Nutricionista e mestra em Saúde, Ambiente e Trabalho pelo Programa de Pós-Graduação em Saúde, Ambiente e Trabalho (PPGSAT) da Universidade Federal da Bahia (UFBA). Doutoranda em Alimentos, Nutrição e Saúde pela Escola de Nutrição da UFBA e pesquisadora do Centro de Integração de Dados e Conhecimentos para Saúde (Cidacs) da Fundação Oswaldo Cruz (Fiocruz) da Bahia.

10 Enfermeira pela Universidade do Estado da Bahia (UNEB), mestre pela Universidade Federal da Bahia (UFBA). Doutora pela London School of Hygiene \& Tropical Medicine (LSHTM). Atualmente, é Sir Henry Wellcome Fellow na LSHTM.

11 Nutricionista pela Universidade Federal do Rio de Janeiro (UFRJ). Mestra em Saúde Pública e Doutora em Administração Pública pela Universidade Federal da Bahia (UFBA). Professora associada da Escola de Nutrição da UFBA.

12 Economista, doutora em Saúde Pública pela Fundação Oswaldo Cruz (Fiocruz). Professora da Escola Fiocruz de Governo, pesquisadora associada à Universidade de Nottingham e consultora internacional em Desenvolvimento e Proteção Social.

13 Médico, mestre em Saúde Comunitária pela Universidade Federal da Bahia (UFBA) e doutor em Epidemiologia pela London School of Hygiene and Tropical Medicine (LSHTM). Professor titular aposentado do Instituto de Saúde Coletiva (ISC) da Universidade Federal da Bahia (UFBA) e pesquisador especialista da Fundação Oswaldo Cruz (Fiocruz) da Bahia.

SILVA, R. de C. R.; PEREIRA, M.; ARAGÃO, E.; GUIMARÃES, J. M. de M.; FERREIRA, A. J. F.; ROCHA, A. dos S.; SILVA, N. J.; TEIXEIRA, C. S. S.; FALCÃO, I.; PAIXÃO, E. S. da; SANTOS, S. M. C.; CAMPELLO, T; BARRETO, M. L. COVID-19 e repercussões na segurança alimentar e nutricional no Brasil. In: BARRETO, M. L.; PINTO JUNIOR, E. P.; ARAGÃO, E.; BARRAL-NETTO, M. (org.). Construção de conhecimento no curso da pandemia de COVID-19: aspectos biomédicos, clínico-assistenciais, epidemiológicos e sociais. 


\section{Introdução}

O aumento dramático no número de pessoas famintas ocorre no momento em que a pandemia da COVID-19 se alastra para regiões nas quais os sistemas de proteção social são historicamente os mais fracos do mundo e vêm sendo ainda mais desmontados com a ascensão de governos com tendência econômica neoliberal. (PARAGUASSU, 2020) A edição do documento The State of Food Security and Nutrition in the World/2020 aponta que quase 690 milhões de pessoas passaram fome em 201910 milhões a mais do que em 2018. (FAO et al., 2020) Os altos custos e baixo acesso aos alimentos significam que bilhões de pessoas não podem se alimentar de maneira adequada e tão pouco têm acesso a uma alimentação variada e saudável. Os famintos são mais numerosos na Ásia, mas se expandem mais rapidamente na África; o que faz este continente a região onde a desnutrição é mais elevada proporcionalmente, em torno de quase 20\%. Na América do Sul, 7,7\% dos domicílios estão em Insegurança Alimentar Grave (IAG). A América do Sul somava 22,5 milhões de habitantes em IAG em 2014, avançando para 31,6 milhões em 2019, um aumento de $44,4 \%$ em 5 anos. $O$ acesso irregular de alimentos também é um desafio para os países de alta renda, atingindo $8 \%$ 
da população na América do Norte e na Europa. Esses resultados permitiram a Food and Agriculture Organization (FAO) afirmar que o mundo não está no caminho de alcançar a meta do Fome Zero até 2030. (FAO et al., 2020)

No Brasil, 68,9 milhões de domicílios 36,7\% (o equivalente a 25,3 milhões) estavam com algum grau de Insegurança Alimentar (IA): IA leve (24,0\%, ou 16,4 milhões), IA moderada $(8,1 \%$, ou 5,6 milhões) ou IA grave (4,6\%, ou 3,1 milhões). (IBGE, 2020) No pais, a desnutrição alcançou até 5,2 milhões de brasileiros no triênio 2015-2017. (PARAGUASSU, 2020) Além da desnutrição, o sobrepeso e obesidade continuam a crescer em todas as regiões do país, particularmente entre adolescentes e adultos. Algumas dessas regiões enfrentam uma epidemia de múltiplas cargas de má nutrição, que combina formas variadas de má nutrição - desnutrição coexistindo com a obesidade e/ou doenças crônicas não transmissíveis relacionadas à dieta. (TEMPONI; VELASQUEZ-MELENDEZ, 2020) A baixa ingestão de micronutrientes como ferro, cálcio e vitaminas A e D, doenças carenciais conhecidas como "fome oculta", denunciada desde os estudos de Josué de Castro nos anos 1940, permanecem sendo importantes problemas nutricionais no país. (FAO et al., 2019; VASCONCELOS, 2008) Essa condição traz sérias implicações para a saúde e o desenvolvimento físico e cognitivo, com efeitos diretos na qualidade de vida das pessoas. A anemia é um dos problemas nutricionais que trazem riscos mais significativos para a saúde, particularmente quando ocorre durante a gravidez. Aproximadamente $27,2 \%$ das mulheres em idade fértil no Brasil (14 a 49 anos) sofriam de anemia. (FAO et al., 2019)

Esse conjunto de dados e informações é relevante neste contexto porque, de uma parte, revelam que a pandemia não poderá ser responsabilizada isoladamente pela severidade que se anuncia na situação de fome, desnutrição e Insegurança Alimentar e Nutricional (ISAN) de todos. Antes, as desigualdades sociais não 
superadas, o avanço de políticas neoliberais e o desmonte do sistema que contemplava políticas sociais inclusivas e promotoras da Segurança Alimentar e Nutricional (SAN) vêm se somando para a situação atual, que tende ao agravamento diante dos impactos da pandemia. Decerto, a insegurança alimentar nos domicílios brasileiros, deve tender à maior magnitude, com o advento da pandemia da COVID-19.

\section{As crises social, econômica e política que antecedem a COVID-19}

Diversas políticas públicas foram implementadas no início deste século que permitiram ao Brasil superar problemas históricos de má nutrição e resultaram na saída do Brasil do Mapa da Fome das Nações Unidas em 2014. (COMISIÓN ECONÓMICA PARA AMÉRICA LATINA Y EL CARIBE, 2020) Uma delas é o Programa Bolsa Família (PBF), um programa de transferência condicional de renda. (BRASIL, 2015) O PBF ajudou o Brasil a cumprir com a meta de redução da pobreza em todos os estados. A transferência de renda diretamente para os beneficiários colaborou para a redução da desigualdade de renda, e as condicionantes acompanhadas pelo PBF permitiram a evolução do Índice de Desenvolvimento Humano (IDH). Estima-se que o componente de transferência de renda adotado pelo programa tenha sido responsável pela retirada de mais de 20 milhões de pessoas da situação de pobreza no Brasil. (DANTAS et al., 2018) Entretanto, essa tendência, vem sendo revertida, com aumento da pobreza e da desigualdade. (SOUZA et al., 2019)

O grupo de pessoas em pobreza extrema no Brasil, que inclui os que vivem com menos de 1,9 dólar por dia, ganhou cerca de 170 mil novos integrantes em 2019 e encerrou o ano passado com 
13,8 milhões de pessoas, o equivalente a 6,7\% da população do país. É o quinto ano seguido no qual o número de brasileiros na miséria cresce. O principal motivo para o aumento do número de brasileiros em pobreza extrema são deficiências na execução do PBF. Em 2019, mais de 1 milhão de famílias estavam aptas a receber o benefício, contudo não foram contempladas pelo programa. (IBGE, [200-])

As políticas sociais com impacto na renda, na pobreza, e na SAN dos brasileiros perderam força desde 2016, com a ascensão de Michel Temer à Presidência da República. Nesse âmbito, o novo governo, que tomou posse em janeiro de 2019, herdou e aprofundou os graves problemas sociais. O desmonte, iniciado em 2016, do Sistema Nacional de Segurança Alimentar e Nutricional (Sisan), criado em 2006, especialmente pela redução de recursos e da cobertura de programas estruturantes, como o represamento dos programas de transferência de renda - PBF e Benefício de Prestação Continuada-BPC -, o Programa de Aquisição de Alimentos da Agricultura Familiar - que desenvolve-se por meio da aquisição de alimentos de agricultores familiares, com mecanismo diferenciado de compras públicas, por valores referenciados pelos mercados regionais -, o Programa Restaurantes Populares - estabelecimento que vende refeições de qualidade a preços reduzidos, geralmente em torno de R\$ 1,00 -, o Programa Água para todos - com as cisternas construídas nas regiões mais pobres do país onde ocorrem longos períodos de estiagem -, entre outros, e a extinção do Ministério de Desenvolvimento Agrário refletem o descompromisso do governo no combate à fome e à miséria no pais. Cabe registrar ainda o fim da política de valorização do salário-mínimo, fundamental para preservar o poder de compra para a população de baixa renda, e a desestruturação da legislação trabalhista - reforma da Consolidação das Leis do Trabalho (CLT) -, que gerou forte aumento da informalidade e na precarização do 
trabalho, ambos com impactos na renda das famílias mais vulneráveis. (ROSSI; MELLO, 2017; SANTARELLI et al., 2017)

Sem dúvida, a aprovação da Emenda Constitucional n ${ }^{\circ}$ 95, em 2016, que congelou os gastos públicos por 20 anos, integra fortemente esse cenário. Esse processo de desmonte afetou não somente o Sisan, como também o Sistema Único de Saúde (SUS) e o Sistema Único de Assistência Social (SUAS), levando ao aprofundamento da situação de pobreza e de vulnerabilidade social vivenciado por muitas famílias brasileiras, indo na contramão do processo da redução da desigualdade social e redistribuição de renda, experimentado no país entre os anos de 2003 e 2014. (NERY, 2019; SANTARELLI et al., 2019)

\section{Desafios econômicos e consumo alimentar no Brasil - contexto da pandemia}

A crise sanitária gerada pela COVID-19, com fortes impactos econômicos e sociais no mundo, atingiu o Brasil num momento de desestruturação do mercado de trabalho, aumento do desemprego e precarização da rede de proteção social. Em consequência disso, as despesas com consumo familiar caíram segundo o Instituto Brasileiro de Geografia e Estatística (IBGE), condição visível entre os mais pobres. (IBGE, [20-]) Nesse contexto, a IA decorrente das restrições de renda - que dificultam o acesso aos alimentos, avança e intensifica no país. A população mais vulnerável, tende a comprar alimentos mais baratos, que geralmente são densos em energia e pobres em nutrientes, para poderem alimentar suas famílias. No entanto, sabe-se que essas pessoas mais vulneráveis à crise de alimentos e nutrição no contexto da COVID-19 são aquelas que vinham sendo expostas a privações alimentares desde antes do início da crise. Ademais, para atender ao chamado do "fique 
em casa", mesmo que mais pessoas estejam cozinhando em casa, os tipos de alimentos que estão sendo comprados e armazenados em estoque são aqueles processados e os ultraprocessados devido à sua maior vida útil; ambos associados ao maior conteúdo de gordura, carboidratos e calorias, o que facilita um maior ganho de peso quando comparados àqueles que compõem as dietas saudáveis e mais equilibradas. (UNIVERSIDADE DE SÃO PAULO, 2020) Assim, os brasileiros mais vulneráveis economicamente estão forçosamente optando por um padrão alimentar de baixa qualidade, escolhendo alimentos mais calóricos, muitas vezes mais baratos, para matar a fome. (THE INTERNATIONAL PANEL OF EXPERTS ON SUSTAINABLE FOOD SYSTEMS, 2020) O fato é que as escolhas alimentares são influenciadas por aspectos individuais e por aspectos ambientais, especialmente o preço.

Como já era esperado, os preços dos alimentos comprados para consumo no domicílio avançaram nessa pandemia. O Índice Nacional de Preços ao Consumidor Amplo 15 (IPCA-15) subiu 0,45\% em setembro, 0,22 ponto percentual (p.p.) acima da taxa de agosto $(0,23 \%)$, e registrou o maior resultado para um mês de setembro desde 2012 , quando foi de $0,48 \%$. A alta no setor foi puxada não só pelas carnes - cujos preços subiram 3,42\% e tiveram o maior impacto entre os alimentos, de 0,09 p.p. -, mas também pelo tomate (22,53\%), óleo de soja (20,33\%), arroz (9,96\%) e leite longa vida (5,59\%). Os três últimos itens acumularam altas de 34,94\%, $28,05 \%$ e $27,33 \%$ no ano, respectivamente. (IBGE, [200-?]) Os preços dos alimentos dispararam devido a:

I. expansão das exportações, já que o preço em dólar é muito favorável e a falta da comoditie no mercado internacional, como resposta de países que fecharam as exportações para proteger seu mercado interno;

II. falta de estoques reguladores e/ou medidas da Companhia Nacional de Abastecimento (Conab) e do governo federal para proteger seu povo. 
A criação da política de estoques reguladores retrocede aos anos 1970, com o propósito de dotar o governo de instrumentos para socorrer os produtores em tempos de safras superabundantes, comprando a produção excedente de forma a amenizar a queda nos preços e assegurar alguma renda ao campo. Em tempos de frustração da produção agrícola, os estoques acumulados nos anos de bonança poderiam ser desovados para, de alguma forma, regular o mercado, evitando volatilidade excessiva e abusos nos preços, combatendo movimentos especulativos e defendendo o consumidor. (DESMONTE..., 2020) Há que considerar ainda que a falta de incentivo, linhas de crédito diferenciadas, e de mecanismos de apoio à comercialização dos produtos da agricultura familiar - responsável por $70 \%$ do fornecimento de alimentos in natura - tem comprometido o escoamento/comercialização de alimentos frescos e saudáveis, o que tem forçado os preços desses produtos para o alto. Como praticamente todos esses fatores continuam sobre a mesa, a perspectivava é que os preços dos alimentos continuem sob pressão nos próximos meses.

Esse conjunto de informações acende um alerta para a qualidade da dieta da população, sobretudo os mais pobres, que buscam alternativas para matar a fome, a exemplo da adoção de práticas inadequados - hiper diluição, redução do tamanho das porções e, consequentemente, dos nutrientes ingeridos, restrição à escolha dos alimentos mais saudáveis e aumento do consumo daqueles mais baratos e pouco nutritivos -, o que aumenta o risco da dupla carga de má nutrição - desnutrição e sobrepeso/obesidade; ocorrendo simultaneamente -, além de outros resultados adversos em saúde. (HUIZAR; ARENA; LADDU, 2020) 


\section{A Segurança Alimentar e Nutricional no Brasil diante da pandemia do novo coronavírus}

A Lei Orgânica de 2006 apresenta um conceito de SAN que consiste na realização do direito de todos ao acesso regular e permanente a alimentos de qualidade, em quantidade suficiente, sem comprometer o acesso a outras necessidades essenciais, tendo, como base, práticas alimentares promotoras de saúde que respeitem a diversidade cultural e que sejam ambiental, cultural, econômica e socialmente sustentáveis. (BRASIL, 2006) A partir de fevereiro de 2010, a alimentação foi incluída entre os direitos sociais previstos no artigo $6^{\circ}$ da Constituição Federal. Entretanto, o Direito Humano à Alimentação Adequada (DHAA) está distante da realidade de muitas pessoas em todo o mundo e, com a pandemia da COVID-19, os desafios são maiores.

De partida, é importante destacar que a fome, em grande parte dos casos, não é uma questão de indisponibilidade de alimentos, mas de insuficiência de renda para adquiri-los. O contexto que já era grave e inaceitável, em pleno início de século XXI - e demandava políticas públicas de caráter emergencial, associadas àquelas estruturantes para enfrentamento da questão ganhou contornos ainda mais dramáticos em função da COVID-19. As medidas de isolamento e distanciamento social tomadas pelo Brasil repercute na condição socioeconômica de inúmeras famílias brasileiras, em especial daquelas em situação de maior vulnerabilidade à ISAN; exatamente a mesma que só poderá cumprir tais medidas se puderem contar com renda auxiliar para ficar em casa. Para suavizar as quedas bruscas do nível de renda e emprego, além de atender aos mais vulneráveis, o congresso aprovou, em abril, a renda básica emergencial de $\mathrm{R} \$ 600$ por pessoa, podendo chegar a R\$1.200 por família, nos casos de mães chefes de família monoparental. Esse auxílio destina-se aos trabalhadores 
informais, autônomos, desempregados, trabalhadores informais e microempreendedores individuais. (BRASIL, 2020a) A medida, inicialmente válida por três meses, foi estendida até o final do ano de 2020 , porém, com redução de $50 \%$ do seu valor; cerca de $44 \%$ dos lares brasileiros receberam auxílio emergencial em agosto, de acordo a Pesquisa Nacional por Amostra de Domicílios (PNAD) COVID-19. (IBGE, 2020) O auxílio emergencial foi popularmente nomeado como "coronavoucher", o que reflete um caráter pejorativo, como algo estigmatizante, e não a perspectiva do direito e dever do Estado para além de uma renda emergencial para os mais vulneráveis; direito que gerou aglomerações nas agências da Caixa Econômica Federal, contrapondo as recomendações da Organização Mundial da Saúde (OMS). (MARCHESAN, 2020)

Um dos programas-chave neste processo de garantia da SAN da população brasileira é o Programa Nacional de Alimentação Escolar (PNAE) do Fundo Nacional de Desenvolvimento da Educação (FNDE), que atende a mais de 40 milhões de alunos das escolas públicas do Brasil. (FNDE, c2017) O fechamento das escolas, medida necessária para conter a propagação do vírus, impôs a interrupção dessa política. Na Resolução $\mathrm{n}^{\circ} 2$, de 9 de abril de 2020, o Ministério da Educação/FNDE, autorizou, a distribuição de gêneros alimentícios adquiridos no âmbito do PNAE às famílias dos estudantes na forma de kits. (BRASIL, 2020b) Infelizmente, o atraso na orientação federal e na coordenação do Sistema Nacional de Educação no que se refere ao PNAE gerou grande discrepância nas iniciativas de governos estaduais e municipais, que tentaram amortecer os impactos da interrupção do fornecimento da alimentação escolar emitindo normas e decretos, para garantir que os recursos ou os alimentos chegassem às famílias carentes e em situação de vulnerabilidade; muitas autoridades locais optaram pela transferência direta de renda, na modalidade de cartão/vale alimentação por se tratar de um meio mais ágil para aumentar 
a renda e garantir a segurança alimentar de famílias que antes contavam com a alimentação escolar para parte dos membros da família, reduzindo os gastos diários com alimentos. Por outro lado, a distribuição dos alimentos permite, também, manter a renda de produtores e fornecedores, inclusive de agricultores familiares. Lembremos que, de acordo com a Lei ${ }^{\circ} 11.947$, de 2009, 30\% do total repassado pelo PNAE deve ser empregado na aquisição de alimentos diretamente da agricultura.

É de nota que os agricultores familiares estão passando por dificuldade para escoar a produção e enfrentam prejuízos financeiros nestes tempos de pandemia. (BORSATTO et al., 2020) A agricultura familiar responde pela maior parte da produção de alimentos destinados ao consumo interno no Brasil. Porém, esse segmento apresenta uma significativa vulnerabilidade econômica, decorrente de diferentes fatores como limitação no acesso a equipamentos que aumentam a produtividade do trabalho, distância das cidades, escala da produção, exploração de atravessadores, entre outros mecanismos. A comercialização de alimentos, sobretudo em feiras livres e mercadinhos, tem sido diretamente afetada pela pandemia, inclusive limitando a renda das famílias agricultoras e camponesas. Portanto, medidas emergenciais de suporte a produção de alimentos precisam ser acionadas, como a criação de seguro emergencial de renda para a agricultura familiar, linhas de crédito diferenciadas e mecanismos de apoio à comercialização, que poderiam mitigar eventuais efeitos negativos no equilíbrio econômico e financeiro dos produtores. Ademais, emerge a necessidade de estabelecimento de cuidados extras com os trabalhadores da agricultura, para que não sejam expostos à COVID-19.

No que se refere à comercialização dos produtos da agricultura familiar, é preciso ampliar iniciativas que permitam a entrega de alimentos, sobretudo de alimentos frescos e saudáveis às pessoas com maiores dificuldades em chegar aos pontos de 
venda; especialmente relevantes para idosos e pessoas em grupo de risco. Em geral, recursos de geoprocessamento permitem identificar locais de produção e comercialização ativos, por tipo de alimentos, de tal forma que consumidores possam seguir consumindo e os produtores possam produzir, comercializar e manter a vida. Decerto, um recurso como esse é útil na emergência, com os comércios e feiras paralisados, mas também pode ser incorporado ao cotidiano pós-pandemia. Algumas plataformas ${ }^{14}$ para esse tipo de apoio foram desenvolvidas a partir das universidades, a exemplo do Laboratório hortas comunitárias (LABNUTRIR) da Universidade Federal do Rio Grande do Norte (UFRN), que faz mapeamento de feiras, hortas e mercados por geoprocessamento e da sociedade civil, como o Mapa colaborativo COVID-19 criado pelo movimento Slow Food Brasil. ${ }^{15}$

Tendo em vista a necessidade de acesso direto aos alimentos ou aos recursos para a obtenção dos mesmos, os restaurantes populares, apesar dos desmontes, têm se organizado e colocado suas estruturas e funcionários para preparar refeições e distribuir para as populações vulneráveis; portanto, sendo estratégicos para assegurar a entrega de refeições acessíveis e nutricionalmente adequadas à população de rua, idosos em situação de pobreza e trabalhadores informais. Acertadamente, alguns desses serviços têm distribuído refeições prontas em bandejas descartáveis para viagem, evitando assim aglomerações nas unidades. Além disso, os horários e dias de distribuição também têm sido ampliados.

Outras iniciativas estão sendo criadas para prover ajuda humanitária às pessoas mais atingidas pela crise econômico-social

14 Ver: nutrir.com.vc.

15 Ver: https://www.google.com/maps/d/u/0/viewer?mid=1CiGBuclqravVTv-

L-L9Uxek9RRyFhQdM\&shorturl=1\&ll=-30.036759299999968\%2C-

$51.21119139999996 \& z=8$. 
causada pela pandemia da COVID-19. As Organizações Não Governamentais (ONGs), igrejas, associações comunitárias e grupos anônimos têm desempenhado papel fundamental no combate à COVID-19, estando na linha de frente, em especial nas favelas e nos territórios onde o poder público não chega, repassando cestas básicas àqueles atingidos pela pobreza e extrema pobreza (exemplo: Ação da Cidadania, uma das maiores e mais respeitadas ONG's do país). Em maio de 2020, o Movimento dos Trabalhadores Sem Terra (MST), dentre outras iniciativas, distribuiu mais de 600 toneladas de alimentos para famílias vulnerabilizadas em todo o país. Partiu também do MST a distribuição de marmitas com produtos agroecológicos para população de rua em algumas capitais, exemplo de Curitiba (PR). (SUDRÉ, 2020)

Particularmente sobre as questões relacionadas à nutrição, é o momento de compartilhar preocupações, alertar e, quem sabe, ampliar o campo de ideias e de práticas para reorientar as pessoas no rumo da boa nutrição. O isolamento social impõe importantes mudanças socioculturais, redução da atividade física e alterações nos hábitos alimentares, determinantes consideráveis do estado nutricional. Evidências convincentes mostraram que os hábitos alimentares são afetados também por condições de angústia e distúrbios emocionais, em que níveis elevados são associados à má qualidade da dieta. Além disso, emoções como medo e tristeza estão associadas a menos desejo ou motivação para comer e menor prazer durante a refeição. (NAJA; HAMADEH, 2020) Portanto, neste momento, importa ser criativo e reforçar políticas públicas para criar alternativas que respeitem o Guia alimentar para população brasileira (2014), recomendado pelo Ministério da Saúde, e que protejam as pessoas da má nutrição.

Em síntese, enquanto ainda há muito a ser conhecido sobre a COVID-19, a influência dessa pandemia na SAN exigirá do poder público, da iniciativa privada e da população ações alinhadas para 
o enfrentamento da situação sem desconsiderar a insegurança alimentar nas suas várias dimensões. Nessa perspectiva, recomendações são apresentadas.

\section{Considerações finais e recomendações}

É importante reafirmar que, em razão dos recentes desafios econômicos, os desempregados e aqueles com perdas generalizadas de renda tendem a comprar alimentos mais baratos, que geralmente são densos em energia e pobres em nutrientes, para poderem alimentar suas famílias. No entanto, sabe-se que essas pessoas mais vulneráveis à crise de alimentos e nutrição no contexto da COVID-19 são aquelas que vinham sendo expostas a privações alimentares antes do início da crise. (ASHBY, 2020) Assim, é possível que os brasileiros mais vulneráveis economicamente estejam forçosamente consumindo padrão alimentar de baixa qualidade, escolhendo alimentos mais baratos para matar a fome. Esse conjunto de dados explicita os riscos de ISAN e má nutrição que já se vinham apresentando, havendo agora a chance de maior aprofundamento pela emergência da epidemia da COVID-19.

Ao desvelar essa situação, urge a necessidade de saídas que coloquem a vida e a dignidade humana no centro das decisões e políticas públicas, salvaguardando os direitos humanos. No caso do DHAA, significa garantir que todas as pessoas, especialmente os mais vulneráveis, tenham acesso a alimentos adequados e saudáveis para poder atender ao clamor de "Fiquem em casa".

O desafio nesse momento é assegurar diferentes mecanismos que contribuam para garantir o DHAA, potencializando diferentes estratégias de abastecimento alimentar. Nesse contexto, o fortalecimento do Programa de Aquisição de Alimentos (PAA) - notadamente as modalidades de compra direta e compra com doação 
simultânea - e a continuidade da operacionalização do PNAE ajustado às demandas sanitárias da COVID-19 - são exemplos de medidas que podem ser rapidamente acionadas. (SAMBUICHI et al., 2020) Políticas de proteção social extraordinárias, como a distribuição de produtos da agricultura familiar, obviamente adaptadas aos cuidados sanitários necessários para reduzir o risco de disseminação do SARS-CoV-2, são de suma importância como estratégia pontual para mitigar a fome de diversos grupos vulneráveis no Brasil. (PEREIRA; OLIVEIRA, 2020; RIBEIRO-SILVA et al., 2020)

Recomenda-se incluir, ainda, iniciativas de educação alimentar e nutricional, por meio de programas educacionais na televisão, virtual ou rádio, que orientem e estimulem a adoção/ manutenção de hábitos alimentares saudáveis para toda a família, incluindo o sempre oportuno estímulo ao aleitamento materno exclusivo até os seis meses de vida, bem como práticas alimentares seguras e saudáveis para crianças menores de dois anos, considerando sempre medidas de precaução sanitária. Neste momento, importa ser criativo para buscar alternativas que disseminem os princípios alimentares e nutricionais e respeitem o Guia alimentar para a população brasileira e que protejam as pessoas da má nutrição. Sem dúvida, além de proteger a renda, as esferas do poder precisam criar um sistema de suporte estruturado e confiável para garantir disponibilidade, acesso, preços acessíveis de produtos alimentares essenciais a todos os membros da família; sistema este crucial para garantir que o alimento seguro e saudável chegue à mesa das pessoas. Em lugares onde os mercados estão em funcionamento, uma modalidade mista de uso de vouchers, juntamente com programas de proteção social, pode ajudar que a comida chegue as famílias mais imediatamente. Não poderia deixar de abordar outro aspecto fundamental para a saúde, que é o acesso à água e ao saneamento, cada vez mais reconhecido como um importante fator protetor em tempos da COVID-19. 


\section{Por fim, reforça-se o fortalecimento do sistema de vigilância e de monitoramento da disseminação do vírus, com vistas a se defi- nirem estratégias alinhadas para o enfrentamento da COVID-19.}

\section{Referências}

ASHBY, N. J. S. The Impact of the COVID-19 Pandemic on Unhealthy Eating in Populations with Obesity. Obesity, Malden, 2020.

BORSATTO, R. S. G. F. et al. Respostas dos municípios para garantir segurança alimentar e nutricional em tempo de pandemia. In: VALENCIO, N.; OLIVEIRA, C. M. de. Crises entremeadas ao contexto de pandemia: antecedentes, cenários e recomendações. São Carlos: UFScar/CPOI, 2020. p. 165-183. Disponível em: https://preprints.scielo.org/index.php/scielo/preprint/view/163/222. Acesso em: 19 jun. 2020.

BRASIL. Lei $n^{\circ} 11.346$, de 15 de setembro de 2006. Cria o Sistema Nacional de Segurança Alimentar e Nutricional - SISAN com vistas em assegurar o direito humano à alimentação adequada e dá outras providências. Diário Oficial da União, Brasília, DF, 18 set. 2006. Disponível em: http://www.planalto.gov.br/ccivil_03/_ Ato2004-2006/2006/Lei/L11346.htm. Acesso em: 19 jun. 2020.

BRASIL. Lei $n^{\circ} 11.947$, de 16 de junho de 2009. Dispõe sobre o atendimento da alimentação escolar e do Programa Dinheiro Direto na Escola aos alunos da educação básica; altera as Leis nos 10.880, de 9 de junho de 2004, 11.273, de 6 de fevereiro de 2006, 11.507, de 20 de julho de 2007; revoga dispositivos da Medida Provisória no 2.178-36, de 24 de agosto de 2001, e a Lei no 8.913, de 12 de julho de 1994; e dá outras providências. Diario Oficial da União, Brasília, DF, 17 jun. 2009. Disponível em: http://www.planalto.gov.br/ccivil_03/_ato2007-2010/2009/lei/ 111947.htm. Acesso em: 18 nov. 2020.

BRASIL. Lei $n^{\circ} 13.982$, de 2 de abril de 2020. Altera a Lei $n^{\circ}$ 8.742, de 7 de dezembro de 1993, para dispor sobre parâmetros adicionais de caracterização da situação de vulnerabilidade social para fins de elegibilidade ao benefício de prestação continuada (BPC), e estabelece medidas excepcionais de proteção social a serem adotadas durante o período de enfrentamento da emergência de saúde pública de importância internacional decorrente do coronavírus (Covid-19) responsável pelo surto de 2019, a que se refere a Lei $n^{\circ} 13.979$, de 6 de fevereiro de 2020. Diário Oficial da União, Brasília, DF, 2 abr. 2020a. 
BRASIL. Ministerio de Desarrollo Social y Combate al Hambre. Brasil Sin Miseria. Organização Tereza Campello, Tiago Falcão e Patricia Vieira da Costa. Brasília, DF, 2015. Disponível em: http://www.mds.gov.br/webarquivos/publicacao/brasil_sem_ miseria/livro_o_brasil_sem_miseria/BSM-espanhol.pdf. Acesso em: 25 maio 2020.

BRASIL. Ministério da Saúde. Departamento de Atenção Básica. Guia Alimentar para a População Brasileira. Brasília, DF: Ministério da Saúde, 2014.

BRASIL. Resolução $n^{\circ}$ 2, de 9 de abril de 2020. Dispõe sobre a execução do Programa Nacional de Alimentação Escolar - PNAE durante o período de estado de calamidade pública, reconhecido pelo Decreto Legislativo $n^{\circ} 6$, de 20 de março de 2020, e da emergência de saúde pública de importância internacional decorrente do novo coronavírus - Covid-19. Diário Oficial da União, Brasília, DF, 13 abr. 2020b.

\section{COMISIÓN ECONÓMICA PARA AMÉRICA LATINA Y EL CARIBE. Panorama}

Social da América Latina. 2020. Disponível em: www.cepal.org/es/suscripciones. Acesso em: 19 jun. 2020.

DANTAS, S. V. de A. D. et al. Uma avaliação do Programa Bolsa Família. Interações, Campo Grande, v. 19, n. 4, p. 713-726, 2018.

(O) DESMONTE de políticas públicas e o avanço da fome entre mais pobres, por Lauro Veiga Filho. Jornal GGN, [s. I.], 22 set. 2020. Disponível em: https://jornalggn. com.br/artigos/o-desmonte-de-politicas-publicas-e-o-avanco-da-fome-entremais-pobres-por-lauro-veiga-filho/. Acesso em: 19 jun. 2020.

FOOD AND AGRICULTURE ORGANIZATION OF THE UNITED NATIONS - FAO et al. The State of Food Security and Nutrition in the World 2020: Transforming food systems for affordable healthy diets. Rome, FAO, 2020.

FOOD AND AGRICULTURE ORGANIZATION OF THE UNITED NATIONS - FAO et al. The State of Food Security and Nutrition in the World: safeguarding against economic slowdowns and downturns. Rome: FAO, 2019.

FUNDO NACIONAL DE DESENVOLVIMENTO DA EDUCAÇÃO (Brasil) - FNDE. Programa Nacional de Alimentação Escolar. Brasília, DF, c2017. Disponível em: https://www.fnde.gov.br/programas/pnae. Acesso em: 10 jun. 2020.

HUIZAR, M. I.; ARENA, R.; LADDU, D. R. The global food syndemic: The impact of food insecurity, Malnutrition and obesity on the healthspan amid the COVID-19 pandemic. Progress in Cardiovascular Diseases, Philadelphia, 2020. 
IBGE. Brasil: indicadores de saúde. Rio de Janeiro, 2020. Disponível em: https:// covid19.ibge.gov.br/pnad-covid/. Acesso em: 2 jun. 2020.

IBGE. Índice Nacional de Preços ao Consumidor Amplo - IPCA. Rio de Janeiro, [200-?]. Disponível em: https://www.ibge.gov.br/estatisticas/economicas/precos-ecustos/9256-indice-nacional-de-precos-ao-consumidor-amplo.html?=\&t=o-que-e. Acesso em: 19 jun. 2020.

IBGE. Pesquisa Nacional por Amostra de Domicílios Contínua - PNAD Contínua. Rio de Janeiro, [200-]. Disponível em: https://www.ibge.gov.br/estatisticas/sociais/ trabalho/9171-pesquisa-nacional-por-amostra-de-domicilios-continua-mensal. html?=\&t=o-que-e. Acesso em: 2 jun. 2020.

IBGE. Rendimento, despesa e consumo. Rio de Janeiro, [20--]. Disponível em: https:// www.ibge.gov.br/estatisticas/sociais/rendimento-despesa-e-consumo.html. Acesso em: 19 jun. 2020.

MARCHESAN, R. Centralizar auxílio de R $\$ 600$ na Caixa criou fila e atraso, dizem analistas. UOL Economia, São Paulo, 6 maio 2020. Disponível em: https://economia. uol. com.br/noticias/redacao/2020/05/06/auxilio-emergencial-problemaspagamento-caixa. htm. Acesso em: 19 jun. 2020.

NAJA, F.; HAMADEH, R. Nutrition amid the COVID-19 pandemic: a multi-level framework for action. European Journal of Clinical Nutrition, London, v. 74, n. 8 , p. $1117-1121,2020$.

NERY, C. Extrema pobreza atinge 13,5 milhões de pessoas e chega ao maior nível em 7 anos. Agência Notícias IBGE. Rio de Janeiro, 7 nov. 2019. Disponível em: https://agenciadenoticias.ibge.gov.br/agencia-noticias/2012-agencia-de-noticias/ noticias/25882-extrema-pobreza-atinge-13-5-milhoes-de-pessoas-e-chega-aomaior-nivel-em-7-anos. Acesso em: 19 jun. 2020.

PARAGUASSU, E. COVID-19, a relação direta entre o capital, solidariedade e as vidas. Brazilian Journal of Implantology and Health Sciences, Macapá, v. 2, n. 3, p. 1-4, Mar. 2020.

PEREIRA, M.; OLIVEIRA, A. M. Poverty and food insecurity may increase as the threat of COVID-19 spreads. Public Health Nutrition, Oxford, p. 1-5, 2020.

RIBEIRO-SILVA, R. de C. et al. Implicações da pandemia COVID-19 para a segurança alimentar e nutricional no Brasil. Ciência e Saúde Coletiva, Rio de Janeiro, v. 25, n. 9, p. 3421-3430, 2020. 
ROSSI, P.; MELLO, G. Choque recessivo e a maior crise da história: a economia brasileira em marcha à ré. Nota do Cecon, Campinas, n. 1, abr. 2017. Disponível em: https://www.eco.unicamp.br/images/arquivos/NotaCecon1_Choque_recessivo_2. pdf. Acesso em: 19 jun. 2020.

SAMBUICHI, R. H. R. et al. O Programa de Aquisição de Alimentos (PAA) como estratégia de enfrentamento aos desafios da COVID-19. Revista de Administração Pública, Rio de Janeiro, v. 54, n. 4, p. 1079-1096, 2020.

SANTARELLI, M. B. V. et al. Trabuco L. Da democratização ao golpe: avanços e retrocessos na garantia do direito humano à alimentação e à nutrição adequadas no Brasil. Brasília, DF: FIAN Brasil, 2017.

SANTARELLI, M. D. G. et al. Informe Dhana 2019: autoritarismo, negação de direitos e fome. Brasília, DF: FIAN Brasil, 2019.

SOUZA, L. E. P. F. de et al. The potential impact of austerity on attainment of the Sustainable Development Goals in Brazil. BMJ Global Health, London, v. 4, n. 5 , p. 1-7, 2019.

SUDRÉ, L. Para combater a "pandemia da fome", MST já doou mais de 600 toneladas de alimentos. Do Brasil de Fato, São Paulo, 12 maio 2020. Disponível em: https://mst.org.br/2020/05/12/para-combater-a-pandemia-da-fome-mst-jadoou-mais-de-600-toneladas-de-alimentos/. Acesso em: 10 jun. 2020.

TEMPONI, H. R, VELASQUEZ-MELENDEZ, G. Prevalence of double burden on malnutrition at household level in four Latin America countries. Revista Brasileira de Saúde Materno Infantil, Recife, v. 20, n. 1, p. 27-35, 2020.

THE INTERNATIONAL PANEL OF EXPERTS ON SUSTAINABLE FOOD SYSTEMS. COVID-19 and the crisis in food systems: symptoms $C$, and potential solutions. Bruxelas: IPES-Food, 2020.

UNIVERSIDADE DE SÃO PAULO. Faculdade de Saúde Pública. $8^{a}$ Webinar: insegurança alimentar e nutricional em tempos de Covid-19. São Paulo, 2020. 1 vídeo (2 $\min 20 \mathrm{~s})$.

VASCONCELOS, F. D. A. G. Josué de castro e a geografia da fome no Brasil. Caderno de Saúde Pública, Rio de Janeiro, v. 24, n. 11, p. 2710-2717, nov. 2008. 